\title{
Spacetime constraints on accreting black holes
}

\author{
David Garofald* \\ Jet Propulsion Laboratory, California Institute of Technology, Pasadena CA 91109
}

\begin{abstract}
We study the spin dependence of accretion onto rotating Kerr black holes using analytic techniques. In its linear regime, angular momentum transport in MHD turbulent accretion flow involves the generation of radial magnetic field connecting plasma in a differentially rotating flow. We take a first principles approach, highlighting the constraint that limits the generation and amplification of radial magnetic fields, stemming from the transfer of energy from mechanical to magnetic form. Because the energy transferred in magnetic form is ultimately constrained by gravitational potential energy or Killing energy, the spin-dependence of the latter allows us to derive spin-dependent constraints on the success of the accreting plasma to expel its angular momentum and accrete. We find an inverse relationship between this ability and black hole spin. If this radial magnetic field generation forms the basis for angular momentum transfer in accretion flows, accretion rates involving Kerr black holes are expected to be lower as the black hole spin increases in the prograde sense.
\end{abstract}

\section{INTRODUCTION}

Magnetized accretion onto rotating black holes forms the basis for models of X-ray binaries (XRBs), active galactic nuclei (AGN) and their smaller counterparts, microquasars. Balbus \& Hawley (1991) showed how the magnetorotational instability (MRI) - discovered independently by Velikhov and Chandrasekhar - could lead to angular momentum transport in differentially rotating MHD flow (Velikhov, 1959; Chandrasekhar, 1961), such as in magnetized accretion onto rotating black holes. For a sufficiently small ratio of magnetic pressure to gas pressure, the highly conducting flow advects the magnetic field with it in a process described as flux-freezing. This means that the magnetic field is subject to being deformed and stretched as determined by the gas motion, producing magnetic connections between spatially distinct regions. If gas at an inner accretion disk radius, $r_{i n}$, is coupled magnetically to gas at an outer radius, $r_{\text {out }}$, the magnetic field transports angular momentum from $r_{\text {in }}$ to $r_{\text {out }}$, decreasing the angular momentum of gas at $r_{i n}$ and increasing the angular momentum of gas at $r_{\text {out }}$. As a consequence of this, gas at $r_{i n}$, which loses angular momentum, spirals inward to smaller radial values and larger circular velocities. Gas at $r_{\text {out }}$, instead, migrates to larger radial values compared to $r_{\text {out }}$, compatible with the angular momentum acquired, and settles in circular orbits with lower circular velocities. As long as inward spiraling gas continues to be magnetically coupled to outward migrating gas, angular momentum is transferred at a greater rate as the coupling distance increases. Due to the energetically less favorable configurations, larger coupling distances are limited, and eventually suffer magnetic reconnection. This study highlights the constraints from energy conservation on coupling distance, ultimately suggesting that general relativity imposes a coupling distance constraint that depends mono- tonically on the spin of the black hole. The monotonic nature of this dependence, suggests that jets and outflows produced in magnetized black hole accretion flows, may bear the signature of black hole spin. Section 2 describes the study and presents the results. Section 3 concludes.

\section{SPIN-DEPENDENCE OF ACCRETION FLOWS}

Under the assumption that the MRI is the basic mechanism behind angular momentum transport, we measure the ability of a thin accretion disk to expel its angular momentum and accrete, by determining the amount of work done to extract the angular momentum that exists over a fixed proper distance between $r_{\text {in }}$ and $r_{\text {out }}$. For a fixed mass density flow between $r_{\text {out }}$ and $r_{i n}$, the angular momentum difference between $r_{\text {out }}$ and $r_{\text {in }}$ must be deposited outward of $r_{\text {out }}$. We assume that the ability to extract that angular momentum difference is the same for all black hole spins and show how this assumption becomes increasingly problematic at higher prograde spin. From conservation laws in the Kerr metric, the angular momentum difference that must be extracted to produce a given accretion rate, increases for fixed proper distance as the black hole spin increases in the prograde sense. A work-kinetic energy argument applied to angular momentum extraction follows, motivating a spin-dependent constraint. We begin by introducing two conserved quantities in the Kerr spacetime that originate from timetranslation and azimuthal angle-invariance that are identified as energy and angular momentum per unit mass for circular geodesic orbits in the equatorial plane of a rotating black hole. From the Kerr metric we have

$$
E=-\epsilon_{\nu} p^{\nu}=\left(1-\frac{2 M}{r}\right) \dot{t}+\frac{2 M a}{r} \dot{\phi}
$$

$$
L=\psi_{\nu} p^{\nu}=-\frac{2 M a}{r} \dot{t}+\frac{\left(r^{2}+a^{2}\right)^{2}-\Delta a^{2}}{r^{2}} \dot{\phi}
$$


where $\epsilon$ and $\psi$ are the two spacetime Killing vectors for Boyer-Lindquist coordinates, $p^{\nu}$ is the 4-momentum, dot implies differentiation with respect to proper time, $M$ and $a$ are the mass and spin parameters, $r$ is the radial coordinate, $t$ is the time coordinate, $\phi$ is the azimuthal angle coordinate, and $\Delta=r^{2}-2 M r+a^{2}$. Replacing $\dot{t}$ and $\dot{\phi}$ in terms of metric components, one obtains the following explicit forms for $\mathrm{E}$ and L (Bardeen et al, 1972).

$$
\begin{gathered}
E=\frac{r^{3 / 2}-2 M r^{1 / 2} \pm a M^{1 / 2}}{r^{3 / 4}\left(r^{3 / 2}-3 M r^{1 / 2} \pm 2 a M^{1 / 2}\right)^{1 / 2}} \\
L= \pm M^{1 / 2} \frac{r^{2} \mp 2 a M^{1 / 2} r^{1 / 2}+a^{2}}{r^{3 / 4}\left(r^{3 / 2}-3 M r^{1 / 2} \pm 2 a M^{1 / 2}\right)^{1 / 2}}
\end{gathered}
$$

with upper sign for prograde orbits and lower sign for retrograde orbits.

As mentioned, we choose a fixed proper distance, R, from the marginally stable circular orbit and determine the energy difference between the marginally stable orbit and the radial location for which the proper distance from the marginally stable circular orbit is R. We show the spin dependence of this energy difference in figure 1 and refer to it as work resulting from the difference in kinetic energy of the gas parcel between $r_{\text {in }}$ and $r_{\text {out }}$. We conclude that if the accretion rate is to remain spinindependent, the gas must lose greater energy per mass density, per unit coordinate time, as the spin becomes more prograde.

Given that it is the MRI behind the transfer of angular momentum and energy between $r_{\text {in }}$ and $r_{\text {out }}$, we motivate the existence of a spin-dependence in the energy transferred from mechanical form in the gas, to magnetic form in the following way. We imagine that two gas parcels, initially adjacent and connected via magnetic field (at some radial position that is intermediate between $r_{\text {out }}$ and $r_{i n}$ ), begin to slowly migrate apart. If the two initially adjacent gas parcels become separated by a proper distance $\mathrm{R}$, as assumed, and their radial velocities are small, the energy transferred from kinetic to magnetic form will roughly be equal to that of figure 1 for the specific spin. Therefore, in order for accretion rates to be spin-independent, the energy in the poloidal component of the magnetic field, must increase with increase in prograde spin. But, magnetic reconnection is progressively more likely to occur before the proper coupling distance becomes $\mathrm{R}$, as the spin increases in the prograde direction. The overall conclusion, thus, is that lower accretion rates are energetically preferred as the spin increases in the prograde direction. One might be tempted to consider the spin dependence of the ability to transport angular momentum by focusing on the work required to extract a fixed amount of angular momentum. In other words, instead of approaching the question by determining the work done over a fixed proper distance, consider the work required to extract a fixed angular momentum difference. Although we claim this not to be the appropriate approach, it is perhaps noteworthy that if one were to calculate work vs. spin this way, the trend would be the same (i.e. that greater work is required to extract that fixed angular momentum difference as the spin increases in the prograde direction). Either way, the direct connection assumed here between the energy difference over a fixed proper distance and the likelihood of magnetic reconnection, is speculative. It could be that magnetic reconnection is related to aspects of the Kerr geometry in other non-trivial ways that are not addressed here. Also, since the constraints discussed here depend on the spin parameter of the black hole, they are purely relativistic, so they produce no Newtonian counterpart to this study.

\section{CONCLUSION}

This work highlights features of spacetime that appear to be intrinsically suited to influence the character of MHD accretion flows. Because MHD accretion flows in AGN are linked to outflows that influence the galactic and intergalactic medium (Kormendy \& Richstone, 1995; Magorrian et al. 1998; Marconi \& Hunt, 2003; Gebhardt et al. 2000; Ferrarese \& Merritt, 2000; Tremaine et al, 2002), black hole spin may produce signatures or even influence the evolution of galaxies (Garofalo, 2009). If the MRI forms the foundation for angular momentum transport in magnetized accretion flows, and, given that its modus operandi is based on magnetically connected spatially separated regions, we have derived constraints that influence the spatial connectivity of such regions. Our suggestion is that the spin dependence of the conservation laws may be reflected in the flow in terms of dynamical constraints. Our analysis in section 2 points to the need for greater conversion of energy to magnetic form in order for the accretion rate to be insensitive to black hole spin. From an energetic viewpoint, then, this suggests that the magnetized flow will be subjected to a spin dependence involving lower accretion rates at higher prograde spin. If MHD turbulence is non-local (e.g. Guan et al., 2009), our analysis applies. We note that accretion rates in GRMHD simulations of adiabatic black hole accretion flows, do in fact decrease as the spin increases in the prograde direction (De Villiers, 2003; McKinney, 2004).

\section{ACKNOWLEDGMENTS}

The research described in this paper was carried out at the Jet Propulsion Laboratory, California Institute of Technology, under a contract with the National Aeronautics and Space Administration. D.G. is supported by the NASA Postdoctoral Program at NASA JPL administered by Oak Ridge Associated Universities through contract with NASA. 


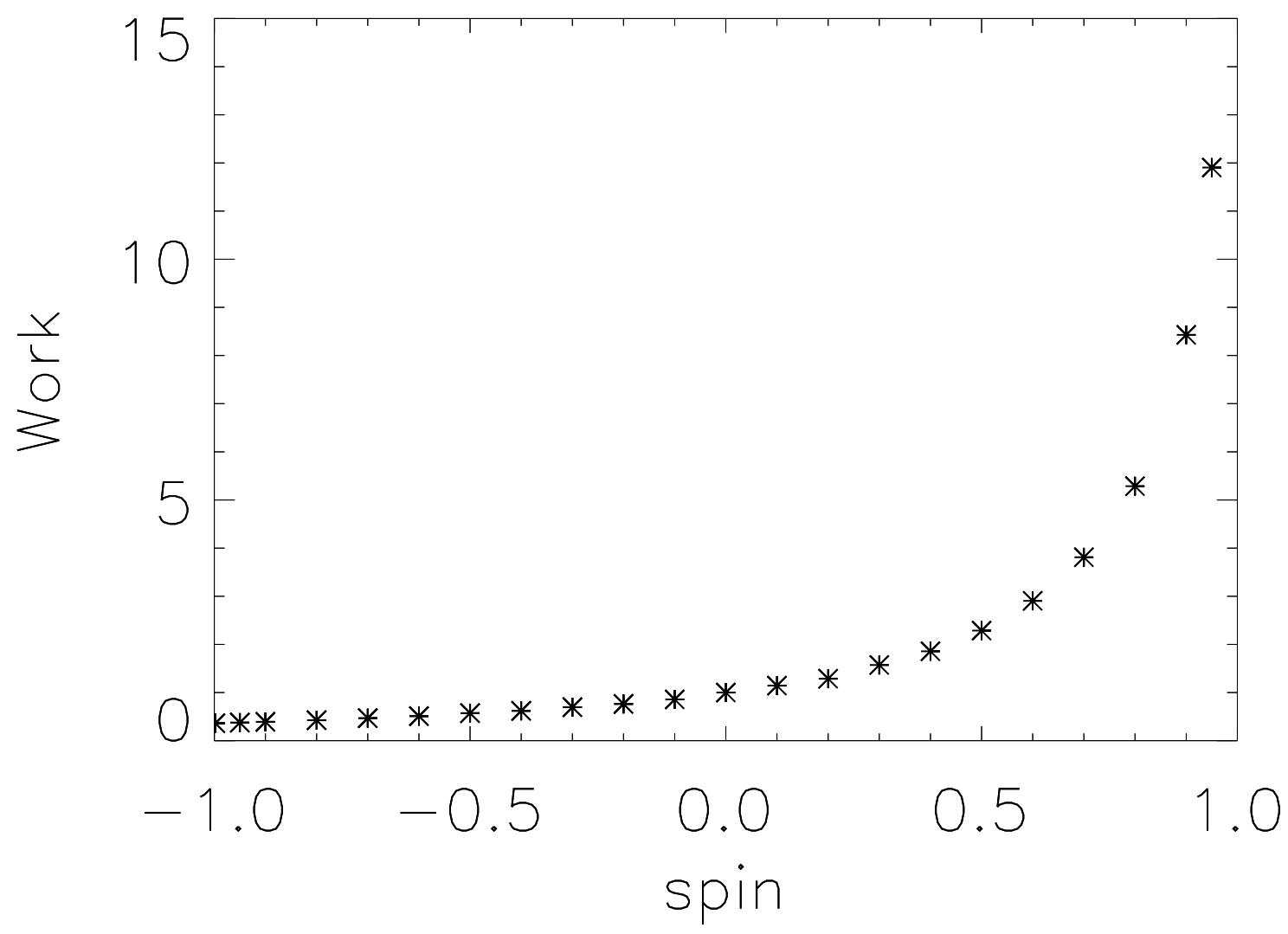

FIG. 1: Work vs. spin illustrating the difficulty in extracting the angular momentum at higher prograde spin. Negative values indicate retrograde accretion while positive ones represent prograde accretion.

\section{REFERENCES}

Balbus, S.A., \& Hawley, J.F., 1991, ApJ, 376, 214

Velikhov, F., 1959, Soviet Phys-JETP, 36, 1398

Chandrasekhar, 1961, Hydrodynamic and Hydromagnetic Stability, Oxford University Press

Bardeen, J.M., Press, W.H., Teukolsky, S.S., 1972, ApJ, 178,347

Kormendy, J., \& Richstone, D., 1995, ARA\&A, 33, 581
Magorrian, J., et al. 1998, AJ, 115, 2285

Marconi, A., \& Hunt, L.K., 2003, ApJ, 589, L21

Gebhardt, K., et al. 2000, ApJ, 539, L13

Ferrarese, L., \& Merritt, D., 2000, ApJ, 539, L9

Tremaine, S., et al 2002, ApJ, 574, 740

Garofalo, D., 2009, ApJL, in press

Guan, X. et al., 2009, ApJ, 694, 1010.

De Villiers, J.P. et al., 2003, ApJ, 599, 1238.

McKinney, J.C., Gammie, C.F., 2004, ApJ, 611, 977 